
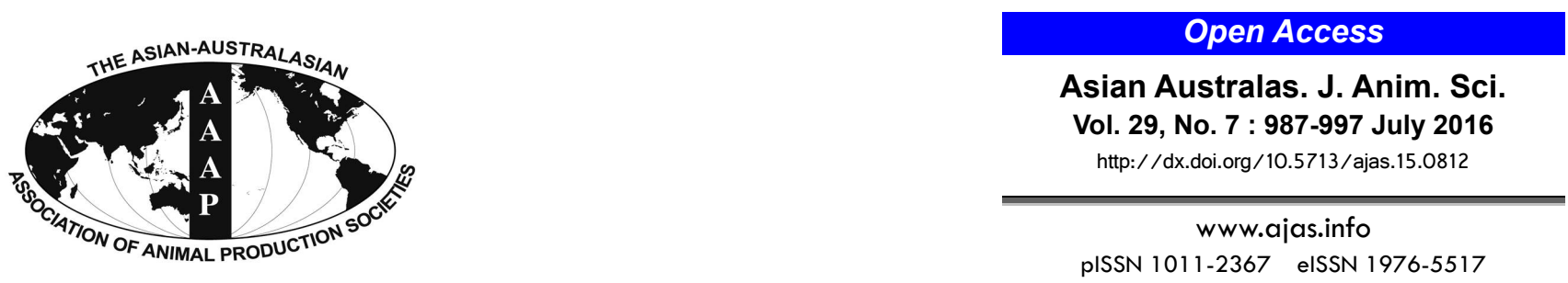

\title{
Comparison of Carcass Characteristics, Meat Quality, and Blood Parameters of Slow and Fast Grown Female Broiler Chickens Raised in Organic or Conventional Production System
}

\author{
Muazzez Cömert*, Yılmaz Şayan, Figen Kırkpınar, Ö. Hakan Bayraktar, and Selim Mert \\ Department of Animal Science, Faculty of Agriculture, Ege University, İzmir 35100, Turkey
}

\begin{abstract}
The objective of the study was to compare the carcass characteristics, meat quality, and blood parameters of slow and fast grown female broiler chickens fed in organic or conventional production system. The two genotypes tested were medium slowgrowing chickens (SG, Hubbard Red JA) and commercial fast-growing chickens (FG, Ross 308). Both genotypes (each represented by 400 chickens) were divided into two sub-groups fed either organic (O) or conventional (C) systems. Chickens of each genotype and system were raised in a semi environmentally controlled poultry house until $21 \mathrm{~d}$ of age and were assigned to 5 pens of 40 chickens each. Then, $\mathrm{O}$ system chickens were transferred into an open-side poultry house with an outdoor run. At $81 \mathrm{~d}$ of age, 10 female chickens from each genotype and from each production system $(n=40)$ were randomly chosen to provide material for analysis, and were weighed and brought to the slaughterhouse to assess carcass characteristics and meat quality. The blood parameters were determined by using 5 female chickens from each genotype and from each production system $(n=20)$. FG had the higher live weight, along with carcass, breast, and thigh-drumstick weights compared to SG $(\mathrm{p}<0.05)$. FG had the higher breast yield, whereas SG had the higher thighdrumstick yield $(\mathrm{p}<0.05)$. The $\mathrm{O}$ system resulted in a higher amount of abdominal fat $(\mathrm{p}<0.05)$. In addition, the $\mathrm{O}$ system values were higher for dry matter, crude ash, crude protein, and $\mathrm{pH}^{15}$ values in breast meat, and for crude ash, crude protein, and $\mathrm{pH}^{15}$ values in drumstick meat $(\mathrm{p}<0.05)$. In addition, total saturated fatty acids, total mono-unsaturated fatty acids, and total omega 3 were significantly higher in the $\mathrm{O}$ system than in the $\mathrm{C}$ system. Thus, the $\mathrm{O}$ system showed a positive advantage compared to the $\mathrm{C}$ system regarding female chicken meat quality, primarily within the ash, protein, and total omega 3 fatty acid profiles. In conclusion, the present study indicated that the main factor affecting the carcass characteristics of female chickens was genotype, whereas the organic system contributed to enhanced meat quality. These findings provide a better understanding of the relative roles of genotype and production systems in female broiler characteristics, and might aid producers in designing their facilities to optimize yield and quality while maintaining acceptable animal welfare standards. (Key Words: Female Broiler, Carcass Quality, Meat Quality, Organic Feeds)
\end{abstract}

\section{INTRODUCTION}

To meet consumer demand for better tasting meat, enhanced animal welfare, and environmental friendly production, new modes of production systems have been established in Europe and the United States (Castromán et al., 2013). To aid producers in making informed decisions about their production systems, a better understanding of the meat quality resulting from widely divergent genotypes

\footnotetext{
* Corresponding Author: Muazzez Cömert. Tel: +90-232-3881867, Fax: +90-232-388-1867, E-mail: muazzez.comert@ege.edu.tr Submitted Oct. 1, 2015; Revised Dec. 31, 2015; Accepted Feb. 10, 2016
}

raised in different production systems and provided different diets is required (Fanatico et al., 2007). Of the many production systems currently in use, the organic rearing production method is generally reputed to reduce stress and increase the overall welfare of the chickens (Castellini et al., 2002a; Fanatico et al., 2005).

Environment and genotype interactions are very important for characteristics of meat quality (Castellini et al., 2002a). However, controversy has arisen with respect to carcass and meat qualitative characteristics regarding whether organic or conventional production results in a better outcome because of the numerous factors involved (Castellini et al., 2002a). Factors that can impact the carcass 
and meat quality of specialty chickens include genotype, age, sex, diet, density, environment, exercise, and pasture intake (Fanatico et al., 2005), because different fast- or slow growing chickens can show different behavior or muscle metabolism (Castellini et al., 2002a). Most authors give priority to the use of slow-growing chickens for organic rearing because of their higher resistance and better adaptation to rearing conditions (Nielsen et al., 2003; Sirri et al., 2010). On the other hand, Castelini et al. (2002a) stated that fast-growing chickens should preferentially be used for organic production primarily for economic reasons even though they are not bred for this type of production, which leads to frequent health and welfare problems. In practice, in the USA fast-growing chickens are primarily used for organic production, whereas European producers tend to use slow-growing genotypes leading to lower body weights at the end of fattening. Because, the fast-growing chickens reach slaughter age after $81 \mathrm{~d}$, as prescribed by European Union regulation (EC Regulation No 889/2008) (Bogosavljević-Bošković et al., 2012).

According to some studies, better meat quality traits, most notably in terms of chemical composition of meat, were observed in organic broilers (Bogosavljević-Bošković et al., 2012). However, other studies have observed no significant effects of production system on the composition of broiler meat (Holcman et al., 2003). In addition, the sex of the bird is also an important factor that affects the carcass composition and meat quality, with some authors stating that the carcass weight and drumstick yield of female chickens are lower than those of male chickens, whereas the breast yield and abdominal fat are much larger (Holcman et al., 2003; Bogosavljević-Bošković et al., 2006).

To address these questions, the objective of the present study was to compare the carcass composition, meat quality (chemical and fatty acid composition), and certain blood parameters of medium slower- and fast-growing female chickens fed using organic and conventional production systems.

\section{MATERIALS AND METHODS}

The trial was conducted at the Poultry Research and Training Center of Ege University from March to June, 2009. All procedures were approved by the internal Ethical Committee of Ege University (Approval No: 2007/51).

\section{Animal materials}

The animal used in the experiment was a total of 40 chickens (10 female chickens for each of two genotypes and two production systems $=4 \times 10$ ) at $81 \mathrm{~d}$ of age. The chickens were randomly chosen from the project titled "The effects of different slaughtering age on performance, carcass and meat quality and some blood parameters of slow-growing and fast-growing broiler chickens taken from conventional poultry enterprises and raised under organic and conventional poultry management system" (Sayan et al., 2010). The two genotypes included medium slow-growing broilers (SG, Hubbard Red JA) and commercial fastgrowing broilers (FG, Ross 308). In the project, a total of 800 mixed gender chicks from two different genotypes were randomly divided into two production systems: fed organic (O) and fed conventional (C). Each system/genotype combination was assigned to 5 pens with 40 chickens.

\section{Organic and conventional systems (barns, lighting, veterinary treatments)}

In designing the study, $81 \mathrm{~d}$, which is the minimum specified according to Turkish Republic Organic Agriculture Regulations (2005/25841) for fast-grown broilers, was determined as the slaughter age of both genotypes. All chicks were obtained from hatcheries complying with hatchery vaccination requirements for Marek's disease, infectious bronchitis, and Newcastle disease. Additionally, Infectious Bursal Disease was given to the chicks with drinking water at wks 2 and 3 of the study.

At the beginning of the study, chicks were individually weighed, wing-banded, and randomly distributed to twenty indoor pens (in five indoor replicates for each genotype with 40 chickens each) in a semi-environmentally controlled poultry house until they reached $21 \mathrm{~d}$ of age. Each $1.4 \times 1.2 \mathrm{~m}^{2}$ floor pen was furnished with wood shaving litter, two round feeders, and two round drinkers. At this point, FG-C and SG-C chickens remained in the same pens (each $2.8 \times 2.4 \mathrm{~m}^{2}$ ) until the end of the study, with three round feeders and two round drinkers. The SG-O and FG-O chickens were transferred into an open side poultry house with outdoor run availability at a minimum of $4 \mathrm{~m}^{2}$ per chicken through a single doorway measuring $50 \mathrm{~cm} \times 70$ $\mathrm{cm}$ for each replicate pen. The open side poultry house pen for each replicate was $3.0 \times 2.5 \mathrm{~m}^{2}$ in size and this floor pen was also furnished with wood shaving litter, three round feeders, and two round drinkers. The vegetation in the outdoor area consisted of $70 \%$ to $75 \%$ gramineae (Hordeum murinum, Bromus tectorum, and Sorghum halepense), 20\% to $25 \%$ leguminosae (Trifolium sp. and Medicago sp.), and $10 \%$ other family plants (Stellaria media, Mercurialis annua, Brassica sp., Malva sylvestris, and Lactuca serriola). Rearing temperatures ranged between approximately $34^{\circ} \mathrm{C}$ and $35^{\circ} \mathrm{C}$ from $1 \mathrm{~d}$ to $7 \mathrm{~d}$, and then it ranged between $27^{\circ} \mathrm{C}$ to $32^{\circ} \mathrm{C}$ from $7 \mathrm{~d}$ to $21 \mathrm{~d}$. After $21 \mathrm{~d}$, if the weather temperature is under $25^{\circ} \mathrm{C}$, the electrical heating system was used to control the temperature at the poultry houses. The lighting program was constant at 23L:1D for the first 2 $\mathrm{d}$ and was decreased to 16L:8D (about $20 \mathrm{~lx}$ ) for rest of the trial. Before the sunset, all organic chickens were collected to the open poultry house and then the artificial lights were 
extinguished closed from 9:30 pm to 5:30 am.

\section{Feeding with organic and conventional systems}

The organic starter and grower diets consisted of $95 \%$ organic feedstuffs. The organic finisher diet was comprised of $98.5 \%$ organic feedstuffs. All organic diets were supplemented with organic maize, soybean meal and wheat. Fish meal and sunflower seed oil were sourced from conventional products for the organic diets. Organic maize, organic soybean meal (organic certificated number by IMOControl-TR = TR-OT-002-İ-0108-1247) and wheat (organic certificated number by IMO-Control-TR = TR-OT-002-İ0108-1355) were obtained from Tiryaki Co. (Gaziantep). All chickens were provided with their assigned diets (Table 1), which included a starter diet (received in the organic diet from 1 to $28 \mathrm{~d}$ and in the conventional diet from 1 to $21 \mathrm{~d}$ ), grower diet (received in the organic diet from 29 to $56 \mathrm{~d}$ and in the conventional diet from 22 to $42 \mathrm{~d}$ ), and a finisher diet (received in the organic diet from 57 to $81 \mathrm{~d}$ and in the conventional diet from 43 to $81 \mathrm{~d}$ ). The included crude protein and metabolizable energy values were based on the recommendations of the production system.

Table 1. Ingredients and nutrient composition of organic and conventional diets

\begin{tabular}{|c|c|c|c|c|c|c|}
\hline \multirow[b]{2}{*}{ Items } & \multicolumn{3}{|c|}{ Organic diet $^{1}$} & \multicolumn{3}{|c|}{ Conventional diet } \\
\hline & $\begin{array}{l}\text { Starter } \\
1 \text { to } 28 \mathrm{~d}\end{array}$ & $\begin{array}{l}\text { Grower } \\
29 \text { to } 56 \mathrm{~d}\end{array}$ & $\begin{array}{c}\text { Finisher } 57 \text { to } \\
81 \mathrm{~d}\end{array}$ & $\begin{array}{c}\text { Starter } \\
1 \text { to } 21 \mathrm{~d}\end{array}$ & $\begin{array}{c}\text { Grower } \\
22 \text { to } 42 \mathrm{~d}\end{array}$ & $\begin{array}{l}\text { Finisher } \\
43 \text { to } 81 \mathrm{~d}\end{array}$ \\
\hline \multicolumn{7}{|l|}{ Ingredients $(\mathrm{g} / \mathrm{kg})$} \\
\hline Maize & 592.70 & 633.75 & 708.62 & 541.43 & 604.64 & 640.53 \\
\hline Soybean meal & 334.31 & 297.39 & 220.00 & 332.59 & 300.95 & 255.41 \\
\hline Wheat & - & - & 39.03 & - & - & 30 \\
\hline Fish meal $^{2}$ & 37.0 & 29.0 & 15.01 & 34.64 & 6.59 & - \\
\hline Sunflower seed oil ${ }^{2}$ & 13.0 & 21.0 & - & 60.0 & 60 & 49.29 \\
\hline Vitamin $\operatorname{mix}^{3}$ & 2.5 & 2.5 & 2.5 & 2.5 & 2.5 & 2.5 \\
\hline Mineral mix & 1.0 & 1.0 & 1.0 & 1.0 & 1.0 & 1.0 \\
\hline DCP-18 & 14.68 & 10.60 & 10.32 & 14.84 & 12.91 & 11.41 \\
\hline $\mathrm{NaCl}$ & 4.81 & 3.59 & 2.95 & 4.26 & 3.25 & 3.32 \\
\hline Marble dust & - & 1.17 & 0.57 & 6.22 & 6.78 & 5.38 \\
\hline DL-methionine, $99 \%$ & - & - & - & 2.52 & 0.95 & 0.66 \\
\hline L-lysine, $98.5 \%$ & - & - & - & 1.34 & 0.43 & 0.50 \\
\hline \multicolumn{7}{|c|}{ Analyzed nutrient composition } \\
\hline $\mathrm{ME}(\mathrm{kcal} / \mathrm{kg})$ & 2898 & 3000 & 2930 & 2957 & 3011 & 3058 \\
\hline Crude protein $(\%)$ & 21.80 & 21.12 & 17.45 & 23.51 & 20.88 & 19.20 \\
\hline Ether extract $(\%)$ & 6.08 & 6.16 & 4.41 & 7.71 & 8.31 & 5.98 \\
\hline Crude cellulose $(\%)$ & 1.57 & 1.91 & 1.14 & 1.08 & 1.39 & 1.19 \\
\hline Total starch, \% & 36.95 & 42.29 & 47.28 & 34.78 & 38.04 & 45.11 \\
\hline Total sugar $(\%)$ & 3.91 & 1.16 & 1.25 & 2.29 & 1.39 & 1.52 \\
\hline Total phosphorus (\%) & 1.20 & 1.09 & 1.01 & 1.04 & 0.89 & 0.94 \\
\hline Calcium (\%) & 5.87 & 5.51 & 5.31 & 5.95 & 5.80 & 4.84 \\
\hline \multicolumn{7}{|l|}{ Fatty acids (g/100 g lipid) } \\
\hline$\Sigma \mathrm{SFA}$ & 12.66 & 17.84 & 14.87 & 15.15 & 13.59 & 15.76 \\
\hline$\Sigma$ MUFA & 22.25 & 26.02 & 27.80 & 27.60 & 27.84 & 27.46 \\
\hline$\Sigma$ PUFA & 59.82 & 48.62 & 53.52 & 52.45 & 55.58 & 52.35 \\
\hline 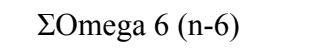 & 55.61 & 45.20 & 51.64 & 50.41 & 53.65 & 50.09 \\
\hline 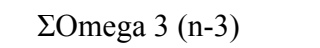 & 4.21 & 3.42 & 1.88 & 2.04 & 1.93 & 2.26 \\
\hline$n-6 / n-3$ & 13.20 & 13.20 & 27.47 & 24.70 & 27.82 & 22.16 \\
\hline
\end{tabular}

DCP-18, dicalcium phosphate; ME, metabolizable energy; $\Sigma$ SFA, total fatty acids; $\Sigma$ MUFA, total monounsaturated fatty acids; $\Sigma$ PUFA, total polyunsaturated fatty acids.

${ }^{1}$ All organic diets were supplemented with organic maize, soybean meal and wheat.

${ }^{2}$ Fish meal and sunflower seed oil were from conventional product for organic diets.

${ }^{3}$ Provided per $2.5 \mathrm{~kg}$ feed of starter diet: vitamin A, 15,000,000 IU; vitamin $\mathrm{D}_{3}, 2,400,000 \mathrm{IU}$; vitamin E, $30,000 \mathrm{mg}$; vitamin $\mathrm{K}_{3}, 5,000 \mathrm{mg}$; vitamin $\mathrm{B}_{1}$, 3,000 mg; vitamin $\mathrm{B}_{2}, 8,000 \mathrm{mg}$; niacin, 25,000 mg; calpan, 12,000 mg; vitamin $\mathrm{B}_{6}, 5,000 \mathrm{mg}$; vitamin $\mathrm{B}_{12}, 20 \mathrm{mg}$; D-Biotin, $50 \mathrm{mg}$; folic acid, 1,000 $\mathrm{mg}$; choline chloride, $400,000 \mathrm{mg}$; vitamin C, 50,000 mg; provided per $2.5 \mathrm{~kg}$ feed of grower and finisher diets: vitamin A, $10,000,000 \mathrm{IU}$; vitamin $\mathrm{D}_{3}$, 1,500,000 IU; vitamin E, 20,000 mg; vitamin $\mathrm{K}_{3}, 3,000 \mathrm{mg}$; vitamin $\mathrm{B}_{1}, 2,000 \mathrm{mg}$; vitamin $\mathrm{B}_{2}, 6,000 \mathrm{mg}$; niacin, 25,000 mg; calpan, 8,000 mg; vitamin $\mathrm{B}_{6}, 4,000 \mathrm{mg}$; vitamin $\mathrm{B}_{12}, 15 \mathrm{mg}$; folic acid, $750 \mathrm{mg}$; choline chloride, 300,000 mg.

${ }^{4}$ Provided per $1 \mathrm{~kg}$ feed for each diet: manganese, 80,000 mg; iron 60,000 mg; zinc, 60,000 mg; copper 5,000 mg; cobalt $200 \mathrm{mg}$; iodine, 1,000 mg; selenium, $150 \mathrm{mg}$. 
Dry matter, crude protein, ether extract, crude fiber, total starch, total sugar, and total phosphorus and calcium were analyzed by Association of Official Analytical Chemists-approved methods (AOAC, 1995). The metabolizable energy content (ME) of the diet was calculated from the chemical composition (McDonald, 2002) as follows $\mathrm{ME} \mathrm{kcal} / \mathrm{kg}=(3.69 \times$ crude protein $)+$ $(8.18 \times$ ether extract $)+(3.99 \times$ starch $)+(3.11 \times$ total sugar $)$.

\section{Carcass composition and organ weights}

At $81 \mathrm{~d}$ of age, 10 female chickens per genotype from each of the two systems were weighed, brought to the slaughterhouse and cut according to the animal welfare law (Anonymous, 2004). Before slaughter, chickens were subjected to a total feed withdrawal of $8 \mathrm{~h}$. The previously weighed chickens were cut using the cervical dislocation method and then plucked and eviscerated. The organ weights (liver, spleen, proventriculus, gizzard, duodenum, ileum and jejunum, cecum, large intestines, heart, bursa fabricus, and pancreas) were recorded. The carcass weight was obtained by removing the head, neck, shanks, and abdominal fat from bled, plucked, and eviscerated chickens. Then, the warm carcass, breast, leg (thigh+drumstick), and abdominal fat weights were recorded. Carcass yields were determined according to the main commercial parts of breast meat (including pectoralis major and pectoralis minor muscles) and the leg (including thigh and drumstick meat), weighed, and the values were expressed as a percentage of carcass weight.

Nutrient composition of breast and drumstick meats and the total fatty acids composition of drumstick meat

The skin and external fat were removed from the breast (pectoralis major) and drumstick meats and then the nutrient composition of these meats (dry matter, crude ash, crude protein, and ether extract) was determined at the University of Ege Animal Science Chemical Analyses Laboratory based on AOAC-approved methods (AOAC, 1995). Samples were pooled by replication and analyzed in duplicate. The $\mathrm{pH}$ values at $15 \mathrm{~min}$ post-slaughter $\left(\mathrm{pH}^{15}\right)$ were measured in each meat samples at three different locations with a digital $\mathrm{pH}$ meter (Hanna Instruments $\mathrm{HI}$ 8314, Padova, Italy) calibrated at $\mathrm{pH} 4.0$ and 7.0. The fatty acid compositions of the diets (Table 1) and drumstick meats were determined on lipids extracted from samples of about $5 \mathrm{~g}$ in a homogenizer with $20 \mathrm{~mL}$ 2:1 chloroform/methanol (Folch et al., 1957). The chloroform phase was removed and evaporated until dry under a vacuum heater set to below $40^{\circ} \mathrm{C}$. The lipid was refluxed with a $1 \mathrm{M}$ solution of potassium hydroxide in $95 \%$ methanol. All lipid samples were then sent to the University of Ege, Central Analytical Laboratory. Total fatty acid compositions were determined by gas-liquid chromatography (Agilent Technologies $6890 \mathrm{~N}$ Network GC System, Anaheim, CA, USA) equipped with a Thermo Scientific TRACE TR-FAME GC Column; $60 \mathrm{~m}, 0.25 \mathrm{~mm}$ ID, $0.25 \mathrm{~m}$ thick (Waltham, MA, USA). Settings were as follows: detector temperature: $250^{\circ} \mathrm{C}$; injection block temperature: $250^{\circ} \mathrm{C}$; Owen temperature: increase gradually from $2^{\circ} \mathrm{C}$ to $225^{\circ} \mathrm{C}$; with helium as the carrier gas. The fatty acids were identified by comparing their retention time and fragmentation pattern with an established standard (SUPELCO-37-Comp. Fame mix $10 \mathrm{mg} / \mathrm{mL}$ in $\mathrm{CH}_{12} \mathrm{Cl}_{2}$ ). The fatty acids were expressed as the percentages of total lipids.

\section{Blood parameters}

Blood samples $(10 \mathrm{~mL})$ were taken from the brachial veins of 5 chickens randomly selected from each genotype and production system, and collected in tubes at $81 \mathrm{~d}$ of age. Blood meant for hematological analysis (white blood cells, red blood cells, hematocrit, hemoglobin, and platelets) were collected into tubes containing EDTA. Blood meant for triode hormone detection (total triiodothyronine, total thyroxin, and thyrotropin-stimulating hormone) were collected into tubes without EDTA. Tubes were centrifuged for $15 \mathrm{~min}$ at 3,000 rpm to obtain a serum that was divided into aliquots for triode hormones and stored in Eppendorf tubes at $-20^{\circ} \mathrm{C}$ until analyses. All blood analyses were performed in a private laboratory using an automatic hematology device (Horiba ABX Pentra-120, Horiba Instruments, West Chicago, IL, USA) for the hemogram values and utilizing a chemiluminescence method with a Roche Cobas device (Roche Diagnostics, Roswell, GA, USA) for measuring triode hormones.

\section{Statistical analyses}

All data were subjected to two-way analysis of variance as a completely randomized design, taking the production system and genotype as main effects, using the SPSS (15.0) for Windows 2006 statistical package (SPPS Inc., Chicago, IL, USA). The statistical model for carcass and organ characteristics $(n=40)$, nutrient compositions of breast and drumstick meat, total and individual fatty acids of drumstick meat $(n=40)$, and some blood parameters $(n=$ 20) utilized the fixed effects of genotype, system, and their interaction. The effects were considered to be significant at $\mathrm{p} \leq 0.05$ and significant differences among the means were determined using Duncan's multiple range tests. All data were expressed as means values with pooled standard errors.

\section{RESULTS}

The results of the study are presented according to the 
Table 2. Carcass characteristics of slow- and fast grown broiler female chickens

\begin{tabular}{|c|c|c|c|c|c|c|c|c|}
\hline & \multicolumn{5}{|c|}{ Carcass composition $(\mathrm{g})$} & \multicolumn{3}{|c|}{ Carcass yield $(\%)$} \\
\hline & $\begin{array}{c}\text { Live } \\
\text { weight }\end{array}$ & $\begin{array}{l}\text { Carcass } \\
\text { weight }\end{array}$ & $\begin{array}{l}\text { Breast } \\
\text { weight }\end{array}$ & $\begin{array}{c}\text { Thigh } \\
+ \text { drumstick } \\
\text { weight }\end{array}$ & $\begin{array}{l}\text { Abdominal } \\
\text { fat }\end{array}$ & $\begin{array}{c}\text { Dressing } \\
\text { out }\end{array}$ & Breast & $\begin{array}{c}\text { Thigh+ } \\
\text { drumstick }\end{array}$ \\
\hline \multicolumn{9}{|l|}{ Treatment } \\
\hline SG-O & $2,781^{\mathrm{c}}$ & $2,067^{\mathrm{c}}$ & $581^{\mathrm{c}}$ & $575^{\mathrm{c}}$ & $80^{\mathrm{ab}}$ & $74.33^{\mathrm{b}}$ & $28.13^{\mathrm{b}}$ & $27.85^{\mathrm{a}}$ \\
\hline FG-O & $4,294^{\mathrm{a}}$ & $3,218^{\mathrm{a}}$ & $1,065^{\mathrm{a}}$ & $823^{\mathrm{a}}$ & $124^{\mathrm{a}}$ & $75.00^{\mathrm{b}}$ & $33.14^{\mathrm{a}}$ & $25.56^{\mathrm{b}}$ \\
\hline SG-C & $2,683^{\mathrm{c}}$ & $2,057^{\mathrm{c}}$ & $538^{\mathrm{c}}$ & $571^{\mathrm{c}}$ & $61^{\mathrm{b}}$ & $76.67^{\mathrm{ab}}$ & $26.22^{\mathrm{b}}$ & $27.73^{\mathrm{a}}$ \\
\hline FG-C & $3,224^{\mathrm{b}}$ & $2,514^{\mathrm{b}}$ & $810^{\mathrm{b}}$ & $710^{\mathrm{b}}$ & $63^{\mathrm{b}}$ & $77.96^{\mathrm{a}}$ & $32.18^{\mathrm{a}}$ & $28.21^{\mathrm{a}}$ \\
\hline SEM & 160 & 124 & 52 & 28 & 9 & 0.44 & 0.71 & 0.32 \\
\hline \multicolumn{9}{|l|}{ Genotype } \\
\hline SG & 2,732 & 2,062 & 560 & 573 & 70 & 75.50 & 27.18 & 27.79 \\
\hline FG & 3,759 & 2,866 & 937 & 766 & 94 & 76.48 & 32.66 & 26.89 \\
\hline \multicolumn{9}{|l|}{ System } \\
\hline $\mathrm{O}$ & 3,538 & 2,643 & 823 & 699 & 102 & 74.67 & 30.64 & 26.70 \\
\hline $\mathrm{C}$ & 2,954 & 2,286 & 674 & 640 & 62 & 77.31 & 29.20 & 27.97 \\
\hline \multicolumn{9}{|l|}{$\mathrm{p}$ values } \\
\hline Genotype & 0.000 & 0.000 & 0.000 & 0.000 & 0.120 & 0.290 & 0.000 & 0.031 \\
\hline System & 0.130 & 0.178 & 0.195 & 0.360 & 0.039 & 0.002 & 0.452 & 0.044 \\
\hline Genotype $\times$ system & 0.000 & 0.000 & 0.000 & 0.000 & 0.035 & 0.013 & 0.000 & 0.001 \\
\hline
\end{tabular}

$\mathrm{SG}$, slow grown; FG, fast grown; $\mathrm{O}$, organic; $\mathrm{C}$, conventional.

Values with different superscripts within a column differ significantly $(\mathrm{p}<0.05)$

effects of the genotype, system, and genotype $\times$ system interactions on carcass characteristics, meat quality, some blood parameters.

\section{Effects of genotype}

The carcass characteristics (live weight, carcass composition, and carcass yield) of SG and FG chickens are presented in Table 2. The effects of the genotype were significant for live weight and all carcass composition and carcass yield measures except for the abdominal fat and dressing out values. FG had higher live weight, carcass, breast, and thigh-drumstick weights compared to SG $(\mathrm{p}<0.05)$. The abdominal fat did not differ statistically between genotypes $(p>0.05)$. Although the effect of the genotype was important for the breast and thigh-drumstick yields $(\mathrm{p}<0.05)$, FG had higher breast yield, whereas SG had higher thigh-drumstick yield $(\mathrm{p}<0.05)$. The organ characteristics of the experimental chickens are presented in

Table 3. Organ characteristic of slow- and fast grown broiler female chickens

\begin{tabular}{lccccccccccc}
\hline & LVR & SPL & P-VEN & GZR & DUE & IL+JE & CE & L-IN & PAN & HRT & BFAB \\
\hline Treatment & & & & & & & & & & & \\
SG-O & $51.37^{\mathrm{b}}$ & $3.56^{\mathrm{ab}}$ & $10.59^{\mathrm{b}}$ & 44.56 & $12.52^{\mathrm{b}}$ & 61.35 & 10.45 & $3.47^{\mathrm{ab}}$ & $4.09^{\mathrm{b}}$ & 9.32 & $4.20^{\mathrm{a}}$ \\
FG-O & $72.39^{\mathrm{a}}$ & $4.27^{\mathrm{a}}$ & $14.25^{\mathrm{a}}$ & 50.79 & $17.66^{\mathrm{a}}$ & 95.77 & 21.72 & $4.26^{\mathrm{a}}$ & $6.13^{\mathrm{a}}$ & 28.84 & $3.10^{\mathrm{ab}}$ \\
SG-C & $38.12^{\mathrm{b}}$ & $3.16^{\mathrm{bc}}$ & $8.90^{\mathrm{b}}$ & 47.22 & $14.24^{\mathrm{ab}}$ & 42.70 & 6.44 & $2.96^{\mathrm{b}}$ & $3.04^{\mathrm{b}}$ & 10.14 & $2.62^{\mathrm{b}}$ \\
FG-C & $46.66^{\mathrm{b}}$ & $2.44^{\mathrm{c}}$ & $8.02^{\mathrm{b}}$ & 42.62 & $17.08^{\mathrm{a}}$ & 118.40 & 8.40 & $2.98^{\mathrm{b}}$ & $3.42^{\mathrm{b}}$ & 14.08 & $2.32^{\mathrm{b}}$ \\
SEM & 3.54 & 0.17 & 0.68 & 1.22 & 0.69 & 11.15 & 2.93 & 0.16 & 4.49 & 0.35 & 0.22 \\
Genotype & & & & & & & & & & & \\
SG & 46.95 & 3.43 & 10.03 & 45.45 & 13.09 & 55.13 & 9.11 & 3.30 & 3.74 & 9.59 & 3.64 \\
FG & 63.81 & 3.66 & 12.17 & 48.07 & 17.47 & 103.31 & 17.28 & 3.83 & 5.23 & 23.57 & 2.82 \\
System & & & & & & & & & & & \\
O & 61.88 & 3.92 & 12.42 & 47.68 & 15.09 & 78.56 & 16.09 & 3.87 & 5.11 & 18.57 & 3.65 \\
C & 42.39 & 2.80 & 8.46 & 44.92 & 15.66 & 80.55 & 7.42 & 2.97 & 3.23 & 12.11 & 2.47 \\
p values & & & & & & & & & & \\
Genotype & 0.014 & 0.498 & 0.113 & 0.292 & 0.001 & 0.028 & 0.167 & 0.106 & 0.029 & 0.122 & 0.069 \\
System & 0.007 & 0.001 & 0.004 & 0.296 & 0.704 & 0.935 & 0.167 & 0.008 & 0.008 & 0.504 & 0.009 \\
$\quad$ Genotype×system & 0.001 & 0.000 & 0.002 & 0.076 & 0.006 & 0.139 & 0.221 & 0.008 & 0.002 & 0.317 & 0.008 \\
\hline
\end{tabular}

LVR, liver; SPL, spleen; P-VEN, proventriculus; GZR, empty gizzard; DUE, duodenum; IL+JE, ileum+jejunum; CE, caeca; L-IN, large intestine, HRT, heart; PAN, pancreas; BFAB, bursa of fabricius; SG. slow grown; FG, fast grown; O, organic; C, conventional; SEM, standard error of the mean.

Values with different superscripts within a column differ significantly $(\mathrm{p}<0.05)$. 
Table 4. Nutrient composition of the breast and drumstick meats of slow- and fast grown broiler female chickens

\begin{tabular}{|c|c|c|c|c|c|c|c|c|c|c|}
\hline & \multicolumn{5}{|c|}{ Breast meat (\%) } & \multicolumn{5}{|c|}{ Drumstick meat (\%) } \\
\hline & $\begin{array}{c}\text { Dry } \\
\text { matter }\end{array}$ & $\begin{array}{c}\text { Crude } \\
\text { ash }\end{array}$ & $\begin{array}{l}\text { Crude } \\
\text { protein }\end{array}$ & $\begin{array}{l}\text { Ether } \\
\text { extract }\end{array}$ & $\mathrm{pH}^{15}$ & $\begin{array}{c}\text { Dry } \\
\text { matter }\end{array}$ & $\begin{array}{c}\text { Crude } \\
\text { ash }\end{array}$ & $\begin{array}{l}\text { Crude } \\
\text { protein }\end{array}$ & $\begin{array}{l}\text { Ether } \\
\text { extract }\end{array}$ & $\mathrm{pH}^{15}$ \\
\hline \multicolumn{11}{|l|}{ Treatment } \\
\hline SG-O & $26.83^{\mathrm{a}}$ & $2.40^{\mathrm{a}}$ & $23.89^{\mathrm{a}}$ & 1.18 & $6.86^{\mathrm{a}}$ & 24.57 & 1.70 & $19.89^{\mathrm{a}}$ & 2.22 & $6.87^{\mathrm{a}}$ \\
\hline FG-O & $26.56^{\mathrm{a}}$ & $2.49^{\mathrm{a}}$ & $23.42^{\mathrm{a}}$ & 2.03 & $6.87^{\mathrm{a}}$ & 25.99 & 1.69 & $19.72^{\mathrm{a}}$ & 3.07 & $6.86^{\mathrm{a}}$ \\
\hline SG-C & $24.17^{\mathrm{b}}$ & $1.10^{\mathrm{b}}$ & $22.95^{\mathrm{ab}}$ & 0.39 & $6.47^{\mathrm{b}}$ & 23.10 & 0.91 & $17.82^{\mathrm{b}}$ & 1.43 & $6.49^{\mathrm{b}}$ \\
\hline FG-C & $24.99^{\mathrm{b}}$ & $1.06^{\mathrm{b}}$ & $21.72^{\mathrm{b}}$ & 0.98 & $6.41^{\mathrm{b}}$ & 25.09 & 0.92 & $17.86^{\mathrm{b}}$ & 3.27 & $6.38^{\mathrm{b}}$ \\
\hline SEM & 0.31 & 0.17 & 0.29 & 0.32 & 0.04 & 0.43 & 0.16 & 0.35 & 0.35 & 0.04 \\
\hline \multicolumn{11}{|l|}{ Genotype } \\
\hline SG & 25.50 & 1.75 & 23.42 & 0.83 & 6.73 & 23.84 & 1.31 & 18.86 & 1.82 & 6.74 \\
\hline $\mathrm{FG}$ & 25.78 & 1.78 & 22.57 & 1.55 & 6.73 & 25.54 & 1.31 & 18.89 & 3.17 & 6.71 \\
\hline \multicolumn{11}{|l|}{ System } \\
\hline $\mathrm{O}$ & 26.70 & 2.45 & 23.66 & 1.60 & 6.87 & 25.28 & 1.70 & 19.81 & 2.65 & 6.86 \\
\hline $\mathrm{C}$ & 24.58 & 1.08 & 22.34 & 0.67 & 6.44 & 24.09 & 0.92 & 17.84 & 2.35 & 6.43 \\
\hline \multicolumn{11}{|l|}{$\mathrm{p}$ values } \\
\hline Genotype & 0.669 & 0.936 & 0.142 & 0.280 & 0.953 & 0.046 & 1.000 & 0.961 & 0.051 & 0.724 \\
\hline System & 0.000 & 0.000 & 0.016 & 0.154 & 0.000 & 0.176 & 0.012 & 0.002 & 0.683 & 0.000 \\
\hline Genotype $\times$ system & 0.001 & 0.000 & 0.030 & 0.362 & 0.000 & 0.108 & 0.117 & 0.027 & 0.228 & 0.000 \\
\hline
\end{tabular}

$\mathrm{SG}$, slow grown; $\mathrm{FG}$, fast grown; $\mathrm{O}$, organic; $\mathrm{C}$, conventional; $\mathrm{pH}^{15}, \mathrm{pH}$ values at 15 min post-slaughter.

Values with different superscripts within a column differ significantly $(\mathrm{p}<0.05)$.

Table 3. The weights of the liver, duodenum, ileum-jejunum, on the total fatty acids composition and on individual fatty and pancreas were significantly affected by genotype, with acids of the drumstick meat was insignificant except for FG having the higher values $(\mathrm{p}<0.05)$. However, the effect $\quad$ C20:6n-6 (Tables 5 and 6 , respectively).

of genotype was important for only the dry matter value of the drumstick meat $(\mathrm{p}<0.05)$ (Table 4), wherein FG had the higher dry matter value. In contrast, the effect of genotype

\section{Effects of system and the genotype $\times$ system interaction}

The system and genotype $\times$ system interaction were

Table 5. Total fatty acids composition of the drumstick meats (g/100 g lipid) of slow- and fast grown broiler female chickens

\begin{tabular}{|c|c|c|c|c|c|c|}
\hline & $\Sigma$ SFA & $\Sigma$ MUFA & $\Sigma$ PUFA & $\begin{array}{c}\text { इOmega } 6 \\
(\mathrm{n}-6)\end{array}$ & $\begin{array}{c}\text { ¿Omega } 3 \\
(\mathrm{n}-3)\end{array}$ & $n-6 / n-3$ \\
\hline \multicolumn{7}{|l|}{ Treatment } \\
\hline SG-O & $27.910^{\mathrm{a}}$ & $37.367^{\mathrm{ab}}$ & $26.522^{c}$ & $24.485^{\mathrm{c}}$ & 2.037 & $12.06^{\mathrm{b}}$ \\
\hline FG-O & $27.018^{\mathrm{a}}$ & $38.477^{\mathrm{a}}$ & $24.866^{\mathrm{c}}$ & $22.959^{c}$ & 1.908 & $12.15^{b}$ \\
\hline SG-C & $23.598^{\mathrm{b}}$ & $32.254^{\mathrm{c}}$ & $36.144^{\mathrm{a}}$ & $34.896^{\mathrm{a}}$ & 1.248 & $28.16^{\mathrm{a}}$ \\
\hline FG-C & $25.610^{\mathrm{ab}}$ & $33.867^{\mathrm{bc}}$ & $31.331^{\mathrm{b}}$ & $29.667^{b}$ & 1.664 & $19.68^{b}$ \\
\hline SEM & 0.614 & 0.935 & 1.413 & 1.499 & 0.125 & 2.240 \\
\hline \multicolumn{7}{|l|}{ Genotype } \\
\hline SG & 25.754 & 34.811 & 29.690 & 31.333 & 1.642 & 20.11 \\
\hline $\mathrm{FG}$ & 26.314 & 36.172 & 26.313 & 28.099 & 1.786 & 15.92 \\
\hline \multicolumn{7}{|l|}{ System } \\
\hline $\mathrm{O}$ & 27.464 & 37.922 & 23.722 & 25.694 & 1.972 & 12.10 \\
\hline $\mathrm{C}$ & 24.604 & 33.061 & 32.281 & 33.737 & 1.456 & 23.92 \\
\hline \multicolumn{7}{|l|}{$\mathrm{p}$ values } \\
\hline Genotype & 0.670 & 0.493 & 0.272 & 0.280 & 0.590 & 0.374 \\
\hline System & 0.011 & 0.003 & 0.000 & 0.000 & 0.030 & 0.002 \\
\hline Genotype $\times$ system & 0.036 & 0.027 & 0.000 & 0.000 & 0.096 & 0.004 \\
\hline
\end{tabular}

SG, slow grown; FG, fast grown; O, organic; C, conventional; $\Sigma$ SFA, total fatty acids; $\Sigma$ MUFA, total monounsaturated fatty acids; $\Sigma$ PUFA, total polyunsaturated fatty acids.

Values with different superscripts within a column differ significantly $(\mathrm{p}<0.05)$. 
Table 6. Drumstick meat individual fatty acids (g/ $100 \mathrm{~g}$ lipid) of slow (SG) and fast grown (FG) broiler female chickens

\begin{tabular}{|c|c|c|c|c|c|c|c|c|c|c|c|}
\hline & $14: 0$ & $16: 0$ & $16: 1 n-7$ & $18: 0$ & $18: 1 n-9$ & $18: 2 n-6$ & $18: 3 n-3$ & $20: 1 n-9$ & $20: 4 n-6$ & $20: 5 n-3$ & $22: 6 n-3$ \\
\hline \multicolumn{12}{|l|}{ Treatment } \\
\hline SG-O & 0.608 & $21.261^{\mathrm{a}}$ & $4.261^{\mathrm{a}}$ & 6.041 & 32.834 & $24.320^{\mathrm{c}}$ & $1.452^{\mathrm{a}}$ & 0.272 & 0.165 & 0.262 & 0.323 \\
\hline FG-O & 0.563 & $20.437^{\mathrm{a}}$ & $4.429^{\mathrm{a}}$ & 6.018 & 33.783 & $22.817^{\mathrm{c}}$ & $1.323^{\mathrm{a}}$ & 0.265 & 0.142 & 0.237 & 0.347 \\
\hline SG-C & 0.476 & $17.265^{b}$ & $2.484^{\mathrm{c}}$ & 5.858 & 29.469 & $34.741^{\mathrm{a}}$ & $0.517^{\mathrm{b}}$ & 0.301 & 0.155 & 0.287 & 0.445 \\
\hline FG-C & 0.507 & $18.988^{\mathrm{ab}}$ & $3.376^{\mathrm{b}}$ & 6.115 & 30.266 & $29.557^{b}$ & $1.072^{\mathrm{ab}}$ & 0.225 & 0.110 & 0.218 & 0.373 \\
\hline SEM & 0.021 & 0.554 & 0.259 & 0.112 & 0.752 & 1.499 & 0.133 & 0.023 & 0.008 & 0.016 & 0.028 \\
\hline \multicolumn{12}{|l|}{ Genotype } \\
\hline SG & 0.542 & 19.263 & 3.372 & 5.950 & 31.152 & 29.531 & 0.984 & 0.287 & 0.160 & 0.275 & 0.384 \\
\hline $\mathrm{FG}$ & 0.535 & 19.713 & 3.902 & 6.066 & 32.024 & 26.187 & 1.198 & 0.245 & 0.126 & 0.228 & 0.360 \\
\hline \multicolumn{12}{|l|}{ System } \\
\hline $\mathrm{O}$ & 0.585 & 20.849 & 4.345 & 6.030 & 33.308 & 23.569 & 1.388 & 0.269 & 0.153 & 0.250 & 0.335 \\
\hline $\mathrm{C}$ & 0.492 & 18.127 & 2.930 & 5.986 & 29.867 & 32.149 & 0.794 & 0.263 & 0.133 & 0.252 & 0.409 \\
\hline \multicolumn{12}{|l|}{$\mathrm{p}$ values } \\
\hline Genotype & 0.880 & 0.705 & 0.330 & 0.625 & 0.587 & 0.285 & 0.450 & 0.390 & 0.034 & 0.160 & 0.704 \\
\hline System & 0.018 & 0.006 & 0.001 & 0.856 & 0.013 & 0.000 & 0.017 & 0.909 & 0.229 & 0.935 & 0.208 \\
\hline Genotype $\times$ system & 0.105 & 0.021 & 0.003 & 0.905 & 0.113 & 0.000 & 0.029 & 0.763 & 0.076 & 0.533 & 0.523 \\
\hline
\end{tabular}

SG, slow grown; FG, fast grown; O, organic; $\mathrm{C}$, conventional.

Values with different superscripts within a column differ significantly $(\mathrm{p}<0.05)$.

important for abdominal fat, dressing out, and thighdrumstick yields (Table 2). The $\mathrm{O}$ system resulted in a higher amount of abdominal fat $(p<0.05)$. The effect of the system was significantly important for the dressing out and the thigh-drumstick yields with the highest values observed in the $\mathrm{C}$ system. The genotype $\times$ system interaction was important for breast yield with higher values obtained for FG-O and FG-C $(\mathrm{p}<0.05)$, and for the live weight and carcass weight with FG-O having higher values of live weight, carcass, breast, and thigh-drumstick weights compared to the other treatments $(p<0.05)$. Furthermore, FG-C had higher values of these parameters than did SG-O and SG-C, between which there were no significant differences (Table 2). The liver, spleen, proventriculus, large intestine, pancreas, and bursa of fabricius values of the chickens were affected by both the system and genotype $\times$ system interactions (Table 3 ). These organ characteristic were higher in the $\mathrm{O}$ system than in the $\mathrm{C}$ system $(p<0.05)$. Finally, the genotype $\times$ system interaction was important for the duodenum with the lowest value observed in SG-O, although this was similar to that of SG-C.

The system and the genotype $\times$ system effects were also found to be important for the dry matter, crude ash, crude protein, and $\mathrm{pH}^{15}$ values in the breast meat, and for the crude ash, crude protein, and $\mathrm{pH}^{15}$ values in the drumstick meat. The $\mathrm{O}$ system values were larger in these criteria than those of the $\mathrm{C}$ system. Additionally, only the $\mathrm{O}$ system positively affected the crude ash values of the drumstick meat $(\mathrm{p}<0.05)$.

The system of $\mathrm{O}$ feed utilized was found to be important for the total fatty acids profiles $(\mathrm{p}<0.05)$ (Table 6$)$. Total saturated fatty acids (SFA), total mono-unsaturated fatty acids (MUFA), and total omega 3 were significantly higher in the $\mathrm{O}$ system than in the $\mathrm{C}$ system, whereas the total poly-unsaturated fatty acids (PUFA), total omega 6 , and $\mathrm{n} 6 / \mathrm{n} 3$ values were significantly elevated in the $\mathrm{C}$ system. The effects of genotype $\times$ system interaction were also important for fatty acids profiles except for the total omega 3 values. The total SFA values of SG-O and FG-O were larger than SG-C and similar to FG-C. The total MUFA value of FG-O was similar to the SG-O value and higher than that of the $\mathrm{C}$ groups. The total PUFA and total omega 6 values of SG-C were the highest values than those of other groups; the SG-O and FG-O values were similar to each other. SG-C had the highest $n 6 / n 3$ values of the drumstick meats compared to the other groups. The effects of the system were important for the C14:0, C16:0, C16:1n-7, C18:1n-9, C18:2n-6, and C18:3n-3 values of the individual fatty acids of the drumstick meat ( $<<0.05)$. The $\mathrm{C}: 14, \mathrm{C} 16: 0$, C16:1n-7, C18:1n-9, and C18:3n-3 values were significantly higher in the $\mathrm{O}$ system than in the $\mathrm{C}$ system, whereas the C18:2n-6 value was significantly larger in the $\mathrm{C}$ system. Furthermore, the genotype $\times$ system interaction was found to be significantly important for the C16:0, C16:1n-7, C18:2n6, and $\mathrm{C} 18: 3 \mathrm{n}-3$ values of the individual fatty acids of the drumstick meat. SG-O and FG-O had the highest values of $\mathrm{C} 16: 0, \mathrm{C} 16: 1 \mathrm{n}-7$, and $\mathrm{C} 18: 3 \mathrm{n}-3$; the former and latter were similar to FG-C. Conversely, SG-C had the lowest value of C16:1n-7 and the highest value of C18:2n-6 compared to the other treatments. The value of C18:2n-6 for FG-C was higher than those of SG-O and FG-O. Finally, neither the genotype nor the genotype $\times$ system interaction affected the 
tested blood characteristics ( $\mathrm{p}>0.05)$ (Table 7).

\section{DISCUSSION}

\section{Carcass composition and organ weights}

At $81 \mathrm{~d}$ of age, the body weight of SG and FG chickens reached 2,732 $\mathrm{g}$ and $3,759 \mathrm{~g}$, respectively. In our study, it appears that the main factor affecting the carcass composition of broilers is genotype, regardless of the production systems used. FG had higher live weight, carcass, breast, and thigh+drumstick weights compared to $\mathrm{SG}$ birds $(\mathrm{p}<0.05)$. The effects of genotype $\mathrm{x}$ system interaction were also found to be important for all carcass characteristics $(p<0.05)$. For example, the live weight of FG-O was higher than that of FG-C $(p<0.05)$. This difference might have primarily been due to the different levels of energy and protein and the feeding phase of both experimental diets as it claimed Kassim and Suwanpradit (1996) and Saleh et al. (1997). In our study, although the starter diet of the $\mathrm{C}$ system had a higher crude protein (CP) content $(+1.71 \%)$, the grower diet of the $\mathrm{C}$ system had lower CP content $(-0.24 \%)$ than the $\mathrm{O}$ system. In addition, the $\mathrm{O}$ system grower diet $(21.12 \%)$ was consumed until experimental day 56, whereas after $42 \mathrm{~d}$, the CP content of the grower diet in the $\mathrm{C}$ system was only $20.88 \%$. However, the energy content of the $\mathrm{C}$ system had an advantage compared to the $\mathrm{O}$ system for all feeding phases: the $\mathrm{C}$ system had a higher energy content of grower, starter, and finisher diets than the $\mathrm{O}$ system, at respectively $59 \mathrm{kcal}, 11$ $\mathrm{kcal}$, and $128 \mathrm{kcal}$. Additionally, the higher live weights of FG-O than FG-C are likely a consequence of the better welfare provided under the O system (Adedeji, 2013) and of the organic feeds themselves, which had different organic acids (Patten and Waldroup, 1988; Castellini et al., 2002a, b). On the other hand, Castelini et al. (2002a) reported that organic Ross broilers had a body weight of $3.614 \mathrm{~kg}$, which was significantly lower than the $4.368 \mathrm{~kg}$ recorded for conventionally reared broilers, whereas BogosavljevićBošković et al. (2011) found no significant differences in body weights. According to the Sales (2014) study, such substantial heterogeneity in carcass composition can arise between studies aimed at examine the comparison of rearing production methods because of the use of different hybrids at different farm locations.

The $\mathrm{O}$ production system resulted in larger abdominal fat values than the $C$ system $(p<0.05)$. Similarly, Poltowicz and Doktor (2011) found that free-range and conventionally reared 42-d-old Ross 308 female broilers had 1.38\% and $0.56 \%$ abdominal fat, respectively. In contrast, Dou et al. (2009) published that the abdominal fat content was generally lower in free-range broilers, and Castelini et al. (2002a) demonstrated that organic Ross broilers showed a considerably lower degree of abdominal fat compared to conventional broilers $(1.0 \%$ vs $2.9 \%, \mathrm{p}<0.01)$, which they suggested was potentially induced by the increased locomotor activity of this breed. According to the recent Sales (2014) meta-analysis study (including 9 studies), access to the pasture in organic and free range systems did not affect abdominal fat values. In addition, Mikulski et al. (2011) proposed that SG had higher abdominal fat value than FG $(2.71 \%$ and $1.74 \%$, respectively), but we did not find any differences between the genotypes.

Many authors have stated that dressing out can be affected by not only genetic factors, but also the outside air

Table 7. Blood parameters of slow- and fast grown broiler female chickens

\begin{tabular}{|c|c|c|c|c|c|c|c|c|}
\hline & $\begin{array}{c}\mathrm{WBC} \\
\times 10^{3} / \mathrm{mL} \\
\end{array}$ & $\begin{array}{c}\mathrm{RBC} \\
\times 10^{6} / \mathrm{mL} \\
\end{array}$ & HcT (\%) & $\mathrm{HgB}$ g/dL & $\begin{array}{c}\text { PLTx } \\
10^{3} / \mathrm{mL} \\
\end{array}$ & $\begin{array}{c}\text { Total T3 } \\
\text { nanogram } / \mathrm{mL}\end{array}$ & $\begin{array}{c}\text { Total } \mathrm{T} 4 \\
\text { microgram } / \mathrm{dL}\end{array}$ & $\begin{array}{r}\mathrm{TSH} \\
\mathrm{mU} / \mathrm{L} \\
\end{array}$ \\
\hline \multicolumn{9}{|l|}{ Treatment } \\
\hline SG-O & 33.6 & 2.47 & 34.8 & 11.07 & 36.0 & 1.78 & 1.42 & 0.008 \\
\hline FG-O & 34.3 & 2.52 & 34.5 & 10.97 & 23.0 & 1.61 & 1.44 & 0.009 \\
\hline SG-C & 33.4 & 2.49 & 34.0 & 10.94 & 35.4 & 1.28 & 1.59 & 0.009 \\
\hline FG-C & 38.2 & 2.37 & 33.2 & 10.32 & 46.2 & 1.36 & 1.21 & 0.010 \\
\hline SEM & 1.71 & 0.03 & 0.52 & 0.14 & 3.50 & 0.08 & 0.06 & 0.001 \\
\hline \multicolumn{9}{|l|}{ Genotype } \\
\hline SG & 33.5 & 2.48 & 34.6 & 11.03 & 35.81 & 1.60 & 1.48 & 0.009 \\
\hline FG & 35.6 & 2.47 & 34.1 & 10.75 & 30.73 & 1.52 & 1.36 & 0.009 \\
\hline \multicolumn{9}{|l|}{ System } \\
\hline $\mathrm{O}$ & 33.9 & 2.50 & 34.7 & 11.02 & 29.81 & 1.71 & 1.43 & 0.009 \\
\hline $\mathrm{C}$ & 35.8 & 2.43 & 33.6 & 10.63 & 40.80 & 1.32 & 1.42 & 0.010 \\
\hline \multicolumn{9}{|l|}{$\mathrm{p}$ values } \\
\hline Genotype & 0.549 & 0.894 & 0.640 & 0.341 & 0.478 & 0.636 & 0.274 & 0.574 \\
\hline System & 0.613 & 0.386 & 0.344 & 0.204 & 0.145 & 0.021 & 0.940 & 0.455 \\
\hline Genotype $\times$ system & 0.603 & 0.37 & 0.775 & 0.833 & 0.153 & 0.115 & 0.257 & 0.841 \\
\hline
\end{tabular}

WBC, white blood cell; RBC, red blood cell; HcT, hematocrit; HgB, hemoglobin; PLT, platelet; T3, total triiodothyronine; T4 total thyroxin; TSH, thyrotropin-stimulating hormone; SG, slow grown; FG, fast grown; O, organic; C, conventional. 
temperature and high quality pasture (Sales, 2014). In our study, although the effect of the genotype was found to be important for the breast and thigh-drumstick yields, the feed system was indicated as playing a larger part in the dressing out values $(p<0.05)$. Specifically, the $C$ system had the highest dressing out values $(p<0.05)$, whereas FG had the biggest breast yield and SG had the biggest thigh-drumstick yield $(p<0.05)$. Similar to our study, in the experiments by Nielsen et al. (2003) and Mikulski et al. (2011), SG chickens were characterized by a significantly $(\mathrm{p}<0.05)$ lower breast muscle yield, but a higher $(p<0.05)$ yield of thigh-drumstick muscles than FG chickens, whereas Bogosavljević-Bošković et al. (2011) observed no effect of free-range production on the percentage of primal carcass cuts including breasts and thighs. In contrast to our study, Castellini et al. (2002a) found differences of $23.2 \%$ vs $22.0 \%$ in breast yield $(\mathrm{p}<0.05)$ between organic and conventional rearing production systems after $56 \mathrm{~d}$. Even larger effects seemed to occur with increasing length of the fattening period up to the organic production standard of 81 days: $25.2 \%$ vs $23.5 \%$, and $15.5 \%$ vs $15.0 \%$ in breast and drumstick yields, respectively $(\mathrm{p}<0.05)$. Furthermore, Sales (2014) stated that the length of the trials and the outside conditions could explain $9 \%$ of the heterogeneity obtained in carcass yield. Finally, the organ characteristic values obtained in the present study were in line with the findings documented by Muriel and Pascual (1995) regarding chickens reared on a free-range.

\section{Nutrient and total meat fatty acids compositions}

The chemical composition of chicken meat, primarily the protein, fat (ether extract), and fatty acid profiles, shows a strong link with the rearing production method (Meluzzi et al., 2009; Mikulski et al., 2011; Bogosavljević-Bošković et al., 2012). In our study, only the dry matter value of drumstick meat was affected by genotype, with FG values being larger than $\mathrm{SG}(\mathrm{p}<0.05)$. The $\mathrm{O}$ system resulted in the biggest values for all important nutrient compositions of breast and drumstick meats $(\mathrm{p}<0.05)$, with the exception of the ether extract values of breast and drumstick meats and the dry matter value of the drumstick meat. Protein content was affected to a much greater degree by the rearing production system than the ether extract content in our study. Similar to our findings, the breast meat of free-range chickens contained significantly more dry matter $(\mathrm{p}<0.01)$ and protein $(\mathrm{p}<0.05)$ than that of chickens raised without outdoor access (Mikulski et al., 2011). On the other hand, Dou et al. (2009) examined slow-growing Gushi chickens under free-range and indoor rearing production systems and found no differences in the fat content $(0.54 \%$ vs $0.86 \%)$ of the meat. Our results were inconsistent with those of Holcman et al. (2003), who found that fattening under indoor and free-range rearing production conditions did not affect the chemical composition of the breast and leg muscle with skin in broilers aged 56 days. In most studies, the differences in the chemical composition of broiler meat can be attributed to broiler foraging activity (Castelini et al., 2002b; Bogosavljević-Bošković et al., 2006) and to natural ambient conditions such as fresh air and sunlight (Bogosavljević-Bošković et al., 2011) because of structural manifestations in both tissues and organs, as well as to their effects on metabolic biochemical processes. Sales (2014) stated that despite no significant differences at the final $\mathrm{pH}$ values of breast muscles, their findings indicated a trend toward increased $\mathrm{pH}$, when chickens had access to pasture. However, we found significant differences between $\mathrm{O}$ and $\mathrm{C}$ for $\mathrm{pH}$, wherein the $\mathrm{pH}^{15}$ values in the $\mathrm{O}$ system were higher than those in the $\mathrm{C}$ system in breast and drumstick meat, at 0.43 and 0.43 , respectively $(p<0.05)$. This situation could be explained by the likelihood that a higher $\mathrm{pH}$ can be correlated with greater movement in the $\mathrm{O}$ system, which improves oxidative metabolism and increases the number of mitochondria in alpha-white fibers and hence converts them into alpha-red fibers. On the other hand, Castellini et al. (2002b) ascribed a lower $\mathrm{pH}$ in organic broilers to better welfare conditions that reduced stress and thus the consumption of glycogen.

Nutrition plays a dominant role in defining the content and ratio of individual fatty acids in poultry meat; therefore, primary attention should be given to the level of pasture availability and fresh plants available for consumption by broilers (Mikulski et al., 2011). In our study, the genotype did not affect the total or individual fatty acids of the drumstick meat, similar to the results shown by Mikulski et al. (2011), wherein 65-d-old chicken genotypes had no impact on breast muscle. However, the total SFA, total MUFA, and total omega 3 were significantly higher in the O system, whereas the total PUFA and omega 6 and n6/n3 values were significantly greater in the $\mathrm{C}$ system in our study. Castelini et al. (2002a) determined similar patterns with respect to SFA and total omega 3 values in the drumsticks; however, their measured PUFA content was not agreement with our results for 81-d-old Ross chickens. Similar to our study, the Sales (2014) study reported that total omega 3 fatty acids showed a positive tendency and the $n=6 / n=3$ values tended to be negative with access to the pasture. Leopold Centre Researchers (2007) suggested that O broilers had a lower proportion of SFA (30.14\%) and MUFA (31.67\%) as compared to C system broilers $(32.31 \%$ and $39.13 \%$, respectively). They also stated that the $\mathrm{O}$ system broilers, on the other hand, had significantly higher levels of PUFA (38.19\% vs 28.57\%) and the values of omega 3 and omega 6 fatty acids (3.92\% and 34.28\%, respectively) were higher in $\mathrm{O}$ broilers as compared to $\mathrm{C}$ broilers $(1.93 \%$ and $26.64 \%)$. On the other hand, our results were inconsistent with those of Kücükyılmaz et al. (2012), 
which found that the total omega 3 value of the thigh meat in SG-O broilers $(2.037 \%$ vs $0.60 \%$ of total lipids) was markedly lower when compared with FG-C broilers (1.664\% vs $1.14 \%$ of total lipids) and SG-C (1.248\% vs $0.78 \%$ of total lipids). In our study, the effects of the system were also important for the C14:0, C16:0, C16:1n-7, C18:1n-9, C18:2n-6, and C18:3n-3 values of the fatty acids of drumstick meat $(\mathrm{p}<0.05)$, and the C14:0, C16:0, C16:1n7, C18:1n-9, and C18:3n-3 values were significantly larger in the $\mathrm{O}$ system than in the $\mathrm{C}$ system, whereas the $\mathrm{C} 18: 2 \mathrm{n}-6$ value was significantly higher in the $\mathrm{C}$ system. Similar results were found by Castellini et al. (2002a) in the drumsticks generated by the $\mathrm{O}$ system: SFA:MUFA:PUFA $=$ $36.18 \%: 31.69 \%: 32.13 \%$ as compared to $\mathrm{C}$ system (34.56\%:37.89\%:27.55\%). Latif et al. (1996), on the other hand, observed that the content of palmitic acid (as the main SFA in the broiler body) was considerably lower in the legs of extensively reared broilers $(22.7 \%)$ than in intensively reared chickens $(26.6 \%)$.

\section{Blood parameters}

Neither the production nor the genotype and nor the genotype $\times$ system interaction affected the measured blood characteristics and thyroid hormone values of chickens $(\mathrm{p}>0.05)$. However, in contrast to our study, Adedeji et al. (2013) stated the existence of management practice effects the hematology (white blood cell and red blood cell) of organic broilers at 12 th week.

In conclusion, the present study indicated that the main factor affecting the carcass characteristics of female chickens, primarily representing live weight, carcass, breast, and thigh-drumstick weights from carcass composition, and breast and thigh-drumstick yields from carcass yield, is genotype. Except for the thigh-drumstick yield values, fastgrown broiler females had higher values of these parameters than did medium slow-grown broiler females. In addition, the $\mathrm{O}$ system positively impacted female chicken meat quality, primarily the ash, protein, and total omega 3 fatty acid profiles compared to the $\mathrm{C}$ system. Together, these findings might aid producers in designing their facilities to optimize yield and quality of female broilers while maintaining acceptable animal welfare standards.

\section{CONFLICT OF INTEREST}

We certify that there is no conflict of interest with any financial organization regarding the material discussed in the manuscript.

\section{ACKNOWLEDGMENTS}

This research was supported by the Technical Research Council of Turkey (TUBITAK Project No. 107 O 696). The authors wish to thank Sakine Uçan for technical assistance.

\section{REFERENCES}

Adedeji, O. S. 2013. Effect of different organic feed ingredients on growth performance, haematological characteristics and serum parameters of broiler chickens. World J. Agric. Sci. 9:137-142.

Anonymous, 2004. Animal Protection (Welfare) Law. Put into force by the Food, Agriculture and Livestock Ministry of Turkey, Official Newspaper (24, June, 2004. No: 5199). Accessed http://www.resmigazete.gov.tr/eskiler/2004/07/20040701.htm\# 2

AOAC. 1995. Official Methods of Analysis, 16th ed. Association of Official Analytical Chemists, Washington, DC, USA

Bogosavljević-Bošković, S., V. Kurćubić, M. D. Petrović, and V. Radović. 2006. The effect of sex and rearing system on carcass composition and cut yields of broiler chickens. Czech. J. Anim. Sci. 51:31-38.

Bogosavljević-Bošković, S., Z. Pavlovski, M. D. Petrović, V. Dosković, and S. Rakonjac. 2011. The effect of rearing system and length of fattening period on selected parameters of broiler meat quality. Arch. Geflügelk. 75:158-163.

Bogosavljević-Bošković, S., S. Rakonjac, V. Dosković, and M. D. Petrović. 2012. Broiler rearing systems: A review of major fattening results and meat quality traits. World's Poult. Sci. J. 68:217-228.

Castellini, C., C. Mugnai, and A. Dal Bosco. 2002a. Effect of organic production system on broiler carcass and meat quality. Meat Sci. 60:219-225.

Castellini, C., C. Mugnai, and A. Dal Bosco. 2002b. Meat quality of three chicken genotypes reared according to the organic system. Ital. J. Food Sci. 14:411-412.

Castromán, G., M. del Puerto, A. Ramos, M. C. Cabrera, and A. Saadoun. 2013. Organic and conventional chicken meat produced in Uruguay: Colour, $\mathrm{pH}$, fatty acids composition and oxidative status. Am. J. Food Nutr. 1:12-21.

Dou, T. C., S. R. Shi, H. J. Sun, and K. H. Wang. 2009. Growth rate, carcass traits and meat quality of slow-growing chicken grown according to three raising systems. Anim. Sci. Pap. Rep. 27:361-369.

Fanatico, A.C., L. C. Cavitt, P. B. Pillai, J. L. Emmert, and C. M. Owens. 2005. Evaluation of slower-growing broiler genotypes grown with and without outdoor access: meat quality. Poult. Sci. 84:1785-1790.

Fanatico, A. C., P. B. Pillai, J. L. Emmert, and C. M. Owens. 2007. Meat quality of slow- and fast-growing chicken genotypes fed low-nutrient or standard diets and raised indoors or with outdoor access. Poult. Sci. 86:2245-2255.

Folch, J., M. Lees, and G. H. Sloane-Stanley. 1957. A simple method for the isolation and purification of total lipides peptides from animal tissues. J. Biol. Chem. 226:497-509.

Holcman, A., R. Vadnjal, B. Žlender, and V. Stibilj. 2003. Chemical composition of chicken meat from free-range and extensive indoor rearing. Arch. Geflügelk. 67:120-124.

Kassim, H. and S. Suwanpradit. 1996. The effects of dietary protein levels on the carcass composition of starter and grower broilers. Asian Australas. J. Anim. Sci. 9:261-266.

Küçükyılmaz, K., M. Bozkurt, A. U. Çatlı, E. N. Herken, M. Çınar, 
and E. Bintaş. 2012. Chemical composition, fatty acid profile and colour of broiler meat as affected by organic and conventional rearing systems. S. Afric. J. Anim. Sci. 42:360368.

Latif, S., E. Dworschák, A. Lugasi, É. Barna, A. Gergely, P. Czuczy, J. Hóvári, M. Kontraszti, K. Neslezlényi, and I. Bodó. 1996. Composition of characteristic components from chickens of different genotype kept in intensive and extensive farming systems. Mol. Nutr. Food Res. 40:319-325.

Leopold Center For Sustainable Agriculture. 2007. A survey commercially available broilers originating from organic, freerange and conventional production systems for cooked meat yields, meat composition and relative value. Leopold Center Progress Report, Ames, IA, USA. pp. 50-53.

McDonald, P., R. A. Edwards, J. F. D. Greenhalgh, and C. A. Morgan. 2002. Animal Nutrition. 6th ed. Pearson Education Limited, Edinburgh Gate, Harlow, Essex, UK. 672 p.

Meluzzi, A., F. Sirri, C. Castelini, A. Roncarati, P. Melloti, and A. Franchini. 2009. Influence of genotype and feeding on chemical composition of organic chicken meat. Ital. J. Anim. Sci. 8:766-768.

Mikulski, D., J. Celej, J. Jankowski, T. Majewska, and M. Mikulska. 2011. Growth performance, carcass traits and meat quality of slower-growing and fast-growing chickens raised with and without outdoor access. Asian Australas. J. Anim. Sci. 24:1407-1416.

Muriel, A. and M. R. Pascual. 1995. Carcass and meat characteristics from free-range chickens. In: Proceedings of the XII European Symposium on Quality of Poultry Meat, Zaragoza, Spain. pp. 219-222.
Nielsen, B. L., M. G. Thomsen, J. P. Sørensen, and J. F. Young. 2003. Feed and strain effects on the use of outdoor areas by broilers. Br. Poult. Sci. 44:161-169.

Patten, J. D. and P. W. Waldroup. 1988. Use of organic acids in broiler diets. Poult. Sci. 67:1178-1182.

Połtowicz, K. and J. Doktor. 2011. Effect of free-range raising on performance, carcass attributes and meat quality of broiler chickens. Anim. Sci. Pap. Rep. 29:139-149.

Saleh, E. A., S. E. Watkins, and P. W. Waldroup. 1997. Changing time of feeding starter, grower, and finisher diets for broilers 3. Birds grown to $3.3 \mathrm{~kg}$. J. Appl. Poult. Res. 6:290-297.

Sales, J. 2014. Effects of access to pasture on performance, carcass composition, and meat quality in broilers: A meta-analysis. Poult. Sci. 93:1523-1533.

Şayan, Y., F. Kırkpınar, Ö. H. Bayraktar, M. Polat, and S. Ünal. 2010. The effects of different slaughtering age on performance, carcass and meat quality and some blood parameters of slowgrowing and fast -growing broiler chickens taken from conventional poultry enterprises and raised under organic poultry management system (In Turkish). Technical Research Council of Turkey (Tubitak) Project Report No. 107 O 696, 77 p. Accessed http://uvt.ulakbim.gov.tr/uvt/index.php?cwid= 9\&vtadi=TPRJ\&ano=136521 52f038f09b3ee850d53e6cd8b77 $4 \mathrm{f} 37 \mathrm{~d}$

Sirri, F., C. Castellini, A. Roncarati, A. Franchini, and A. Meluzzi. 2010. Effect of feeding and genotype on the lipid profile of organic chicken meat. Eur. J. Lipid Sci. Technol. 112:994-1002. SPSS for Windows. 2006. Released 15.0 version, SPPS Inc., Chicago, IL, USA. 